\title{
XXXVI. \\ Ueber die Siedepunkte isomerer Aether von der Formel $\Theta_{n} H_{2 n} \theta_{2}$.
}

Von

\author{
Alfred Wanklyn. \\ (Journ. of the chemic. society. Ser. 2, vol. III. p. 30).
}

Nach Kopp's Arbeit müsste es scheinen, als ob alle untereinander isomere Aether der Fettsäuren, oder alle Aether von der Formel $\boldsymbol{\epsilon}_{n} \mathrm{H}_{2 n} \boldsymbol{\theta}_{2}$, wo $n$ eine Constante ist, denselben Siedepunkt haben müssten, eine Annahme, welche sammt ihren Consequenzen in die Lehrbücher (von Kekulé und von Limpricht) aufgenommen worden ist.

Gelegentlich der Darstellung von baldriansaurem Aethyl und essigsaurem Amyl, welche beide dieselbe Formel $\epsilon_{7} \mathrm{H}_{14} \boldsymbol{\theta}_{2}$ haben, bemerkte ich eine beträchtliche Differenz in den Siedepunkten beider, das erste siedet bei $133^{\circ}$, das letztere bei $140^{\circ}$. Für das essigsaure Amyl fand Cahours $125^{\circ}$, wahrscheinlich war aber sein Präparat noch mit Amylalkohol verunreinigt, denn ein Gemenge beider Körper mit etwas Wasser würde sìch durch Elementaranalyse nicht von reinem essigsauren Amyl unterscheiden lassen.

Das für die obigen Bestimmungen verwandte Präparat war frei von Amylalkohol und um der Reinheit sichrer zu sein, wurde noch eine alkalimetrische Analyse vorgenommen.

Aus dieser und mehreren anderen ähnlichen Thatsachen geht hervor, dass Kopp's Gesetz über die Siedepunkte nicht richtig ist. Die Glykole haben bewiesen, dass bei homologen Körpern nicht jedesmal mit der Zunahme im Atomcomplex auch der Siedepunkt entsprechend steigen müsse, und bei genauer Prüfung fast einer jeden homologen Reihe stellt es sich heraus, dass das Steigen nicht in allen Theilen einer und derselbe Reihe gleich viel beträgt. So siedet z. B. Jodmethyl bei $43^{0}$, Jodäthyl bei $73^{\circ}$, 
270 Broughton: Neue Art der Darstell. von Anhydriden u. Aethern.

und Jodamyl bei $145^{\circ}$, was für den ersten Zuwachs von $\mathrm{CH}_{2}$ ein Steigen von $30^{\circ}$, und von $\frac{73^{\circ}}{3}$ oder $24,3^{\circ}$ für jedes der folgenden $\mathbf{E H}_{2}$ ergiebt.

Weiter siedet Zinkmethyl bei $46^{\circ}$, Zinkäthyl bei $119^{\circ}$ : Differenz für $\mathrm{CH}_{2}$ also $73^{\circ}$, Zinkamyl hingegen siedet bei $220^{\circ}$, Differenz für $\in \mathrm{H}_{2}$ demnach $\frac{100^{\circ}}{3}$ oder $33,7^{\circ}$.

Ausserdem siedet der Holzgeist bekanntlich bei $65^{\circ}$, während K,o pp's Theorie $59^{\circ}$ oder $60^{\circ}$ erfordert.

Weiter hinauf in der Alkoholreihe kommen wir auf Würtz isomere Amylalkohole mit verschiedenen Siedepunkten und dann auf die isomeren Hexylalkohole. Wenn man auch einwerfen kann, dass Amylenhydrat und $\beta$ Hexylalkohol nicht wahre Homologen des Aethylalkohols sind, so beweisen die unerwarteten Siedepunkte dieser Körper doch, dass aus der blosen Formel eines Alkohols sich sein Siedepunkt nicht berechnen lässt.

\section{XXXVII. \\ Ueber eine neue Art der Darstellung von Anhydriden und Aethern.}

Von

John Broughton.

(Journ. of the chem. society. Ser. 2. vol. III. p. 21).

Die ursprünglich von Gerhardt entdeckten Reactionen, zur Darstellung der Anhydride einbasischer Säuren gründen sich entweder auf die gegenseitige Zersetzung des Chlorids eines elektronegativen Radicals mit dem entsprechenden Alkalisalze, unter Bildung des Alkalichlorids und des Anhydrids, oder auf die Einwirkung von Drei-oder Fünffachchlorphosphor auf das Alkalisalz. 